\title{
Sobre os filarideos dos crocodillos sul-americanos (")
}

pelo

\author{
DR. LAURO TRAVASSOS
}

(Com as estampas XXXI--XXXIV).

$\mathrm{Na}$ litteratura helminthologica existe apenas referencia a uma especie de Filarioidea parasitando a crocodillos da America do Sul. Trata-se da Filaria bacillaris Molin, 1858.

Na collecção helminthologica do Instituto Oswaldo Cruz por nós reunida, encontramos duas especies de nematodeos Filarioidea que passamos. a estudar.

E' de notar que a systematica dos Filarioidea acha-se ainda embryonaria havendo um grande numero de generos baseados em especies mal estudadas. Além disto é evidente que os nematodeos reunidos sob a rubrica de Filarioidea têm origens philogeneticas diversas e muito provaveimente este grupo terá de ser desdobrado pelo menos em duas superfamilias. Para uma revisão geral dos Filaroidea torna-se preciso um estudo methodico e acurado de um grande numero de fórmas, o que ha já algum tempo vimos fazendo. Neste nosso trabalho nos limitaremos a descrever as duas fórmas que encontrámos nos jacarés brasileiros. A primeira especie se refere evidentemente ao parasio descripto por Molin, sob o nome de $\mathrm{Fi}$ laria bacillaris e para o qual estabelecemos um novo genero; a segunda, do genero Micropleura ainda não era descripta.

Oswaldofilaria n. g. Filarioidea: Cuticula lisa; bocca simples, seguida de reduzida capsula buccal; esophago extremamente longo, differenciado em uma porção anterior ou pharynge. Femeas opistodelphas; vulva situada pouco acima do meio do corpo; ovejector muito longo e simples; extremidade caudal digitiforme; viviparas; machos com a extremidade posterior digitiforme; sem azas lateraes e com 7 pares de papillas muito nitidas; espiculos curtos, fortes e de tamanho e fórma diversos.

habitat: Paredes thoracicas de crocodillos.

ESPECIE TYPO: Oswaldofilaria bacillaris (Molin, 1858).

(*) Recebido para publicaçâo a 12 de Abril de 1933. 
Este genero approxima-se de Thaumugadia do qual se afasta pela extructura do esophago, posição da vulva e presença de papillas caudaes do macho. Approxima-se de Conospiculum Pandit, Pandit \& Iyer, pela posição da vulva, afastando-se pela extructura do esophago.

Oswaldofilaria bacillaris (Molin, 1858).

(Figs. 1 a 6 ).

Filaria bacillaris Molin, 1858, p. 379.

Filaria bacillaris Diesing, 1861, p. 701.

Comprimento: femeas $45 \mathrm{~mm}$., machos $20 \mathrm{~mm}$; largura: femeas $0,25 \mathrm{~mm}$., machos $0,21 \mathrm{~mm}$.

Cuticula lisa, corpo cylindrico com a extremidade anterior ligeiramente entumecida, mas sem azas lateraes. Extremidade posterior digitiforme nos dois sexos. Bocca núa guarnecida por diminutas papillas das quaes as lateraes são bem apparentes. A bocca conduz a uma capsula buccal de secção longitudinal trapezoidal, porém muito pequena; méde nas femeas cerca de $0,025 \mathrm{~mm}$. de largura por $0,015 \mathrm{~mm}$. de profundidade e nos machos 0,016 por $0,008 \mathrm{~mm}$. O esophago extremamente longo se divide nitidamente em uma porção anterior de cerca de $1 \mathrm{~mm}$. nas femeas e $0,65 \mathrm{~mm}$. nos machos para umia porção posterior de cerca de $8 \mathrm{~mm}$. nas femeas e $4,6 \mathrm{~mm}$. nos machos, isto é, nas femeas o esophago attinge um comprimento total de $9 \mathrm{~mm}$. e nos machos de $5,3 \mathrm{~mm}$. Annel nervoso ao nivel da porção média do esophago anterior, isto é, a cerca de $0,6 \mathrm{~mm}$. da extremidade anterior, nas femeas e $0,36 \mathrm{~mm}$. nos machos.

Femea com extremidade posterior digitiformie, rombuda, ficando o anus situado a cerca de $0,35 \mathrm{~mm}$. da extremidade, vulva situada a cerca de 13 a $21 \mathrm{~mm}$. da extremidade anterior, isto é a cerca de $10 \mathrm{~mm}$. abaixo da terminação do esophago, perto do meio do corpo. Ovejector simples, longo e dirigido de deante para traz; é constituido por um esphincter terminal de cerca de $0,3 \mathrm{~mm}$. de comprimento seguido de um tubo muscular extremamente longo que se transforma progressivamiente em longo vestibulo. Os uteros são dispostos longitudinalmente formando alças pouco accentuadas. Um dos ramos uterinos forma alças que attingem anteriormente a uma distancia de cerca de $1 \mathrm{~mm}$. da extremidade. Posteriormente o utero ultrapassa o anus ficando a uma distancia de cerca de 0,05 $\mathrm{mm}$. da extremidade. Ovarios situados posteriormiente, isto é, as femeas são do typo opistodelpho. Uteros repletos de microfilarias.

Machos com a extremidade posterior enrolada em espiral e terminando em ponta obtusa, digitiforme. Cloaca a cerca de $0,14 \mathrm{~mm}$. da ex- 
tremidade. A abertura cloacal é simples, existindo lateralmente 7 pares de papillas muito nitidas, mas sem azas lateraes.

Papillas equidistantes, sendo dois pares pré-anaes, 3 adanaes e 2 post-anaes. Espiculos simples, relativamente curtos e sem gubernaculo, de dimensões e fórmas differentes; o maior mede cerca de $0,38 \mathrm{~mm}$. de comprimento por cerca de $0,042 \mathrm{~mm}$. de largura mledia e o menor mede cerca de $0,18 \mathrm{~mm}$. de comprimento por uma largura média de $0,042 \mathrm{~mm}$. O maior espiculo é falcado sendo o menor de fórma caracteristica.

habitat: Paredes do thorax de Caiman sclerops Gray.

O material por nós estudado provém dos arredores do Rio de Janeiro.

Molin encontrou esta especie em Champsa nigra e Caiman sclerops.

Micropleura v. Linstow, 1906.

Este genero foi estabelecido para um parasito de Gavialis gangelicus estudado por $\mathbf{v}$. Linstow e posteriormente por Baylis e Daubney. Baylis \& Daubney separam este genero em sub-familia independente, mas attribuiram-no á familia Filariidae. Yorke \& Maplestone consideram tambem como fazendo parte da familia Filariidae.

Pelo estudo que fizemos da especie parasita dos crocodillos Sul-americanos julgamos mais acertado incluil-o na familia Philometridae Baylis \& Daubney, 1926.

A familia Filometridae afasta-se muito dos filarideos senso estricto nos parecendo razoavel deva contistuir um grupo isolado - Philometroidea - ao qual talvez se possa juntar os Dracunculidae. E' fóra de duvida que Philometra e Micropleura apresentam grandes affinidades embora possam ficar em duas sub-familias de uma mesma familia. O genero Conospiculum do qual foi incluido na subfamilia dos Micropleurinae, não deve entrar nesta familia. A' diagnose generica dada por Yorke \& Maplestone faremos as seguintes alterações: Cauda da femea conica; uleros divergentes e sacciformes, vulva atrophiada no adulto; gubernaculo presente ou rudimentar.

\section{Micropleura vazi $\mathrm{n}$. sp.}

(Figs. 7 - 13)

Comprimento: femeas $25 \mathrm{~mm}$; machos $6 \mathrm{~mm}$. Largura: femeas 1 mm.; machos 0,7 $\mathrm{mm}$.

Cuticula lisa com bossas longitudinaes lineares e estriadas transver- 
salmente, distribuidas por toda a superficie do corpo; medem cerca de 0,040 a $0,064 \mathrm{~mm}$. de comprimento. Musculatura polimiaria com campos lateraes estreitos. Dimorphismo sexual accentuado. Bocca simples, parecendo ser primitivamente bilabiada, é guarnecida por 3 pares de papillas muito reduzidas. Póro excretor ao nivel da parte média do esophago, é constituida por volumosa cellula apresentando 4 prolongamentos, dois anteriores e dois posteriores e que se dirigem obliquamente para os campos lateraes; dista o póro excretor cerca de $0,9 \mathrm{~mm}$., nas femeas, e $0,28 \mathrm{~mm}$., nos machos, da extremidade anterior. Bocca sem capsula buccal. Esophago diffęrenciado em uma porção anterior mais delgada, que nas femeas. mede cerca de $0,60 \mathrm{~mm}$. e nos machos $0,33 \mathrm{~mm}$. Na parte posterior desta porção do esophago encontra-se o annel nervoso. A porção posterior do esophago mede cerca de $1,6 \mathrm{~mm}$. nas femeas (comprimento total do esophago $2,2 \mathrm{~mm}$.) e $0,93 \mathrm{~mm}$. nos machos (comprimento total do esophago $1,2 \mathrm{~mm}$.). $\mathrm{O}$ esophago além da differenciação nitida em um esophago muscular ou pharynge e esophago glandular, apresenta em cada uma destas partes um vestigio de separação na parte média de tal modo cada porção é constituida por duas porções sub-iguaes.

Femeas com a extremidade posterior conica, distando o anus cerca de $0,5 \mathrm{~mm}$. da extremidade; vulva punctiforme, situada na parte media do corpo. Ovejector muito reduzide, atrophiado nas femeas gravidas. Utero constituido por um amplo sacco fusiforme e sem formar alcas, tendo na parte média o ovejector (semelhante ao que se observa em Rhabdias). Oviductos curtos bem como os ovarios. Ovarios situados nas extremidades do corpo (typicamente amphidelphos) e terminando em uma volumosa cellula resfringente (fiig. 8). No utero encontram-se todas as fórmas evolutivas das larvas sem a formação de ovos typicos. As morulas e as larvas (fig. 11) novas são envolvidas por delicada membrana difficil de perceber. $\mathrm{Na}$ evolução das larvas, no utero, percebe-se desde os primeiros estadios (fig. 11) uma extremidade mais grossa e que se tinge mais fortemente pelo carmim, extremidade cephalica. As larvas completamente desenvolvidas são subuladas posteriormente e medem cerca de $0,37 \mathrm{~mm}$. de comprimento por $0,013 \mathrm{~mm}$. de largura maxima. Apresentam vestigio do tubo digestivo já differenciado numa porção anterior ou esophago de cerca de $0,11 \mathrm{~mm}$. de comprimento e uma posterior de cerca de $0,13 \mathrm{~mm}$. o anus dista da extremidade posterior que é subulada cerca de 0,10 a $0,14 \mathrm{~mm}$. Ao nivel da parte média da porção esophagiana existe uma curta faixa transversal clara e não se deixando corar, que interpretamos como primordio da grande cellula excretora. Esta mancha fica a cerca de $0,067 \mathrm{~mm}$. da extremidade anterior. Sendo o ovejector atrophiado, no adulto, a eliminação das larvas se dará pela ruptura das paredes do utero e do corpo, o que 
aliás é justificado pela fragilidade destas paredes que se rompem expontaneamente, quando os exemplares gravidos não são fixados, logo após serem retirados do hospedador.

Machos com a extremidade posterior curvada em espiral e apresentando estreitas azas lateraes, sustentadas por 7 pares de papillas dispostais do modo seguinte: 4 pares post-anaes, mais ou menos equidistantes; 3 pares pre-anaes tambem equidistantes.

Existe tambem uma papilla impar logo acima da cloaca. Na face ventral existem duas series de bossas cuticulares que lembram a formação descripta por Baylis, para a $M$. vivipara. Espiculos delgados, filiformes, sub-iguaes, medem cerca de $0,168 \mathrm{~mm}$. de comprimento. Gubernaculo presente, cuneiforme, mede cerca de $0,056 \mathrm{~mm}$. de comprimento. Anus a cerca de $0,29 \mathrm{~mm}$. da extremidade posterior. Tubo genital macho constituido de um canal ejaculador de cerca de $0,73 \mathrm{~mm}$. de comprimento e, de um tubo testicular propriamente dito, sub-rectilineo e indo terminar adiante do fim do intestino.

habitat : Cavidade abdominal de Caiman sclerops Gray.

Fazendo uma revisão em nossas necropsias verificámos havermos examinado 20 exemplares de Caiman sclerops distribuidos do modo seguinte:

4 exemplares provenientes dos arredores do Rio de Janciro dos quaes 2 estavam parasitados com $M$. vazi e um com Oswaldofilaria bacillaris.

8 examinados em Matto Grosso (Rio S. Lourenço) dos quaes 3 estavam parasitados com $M$. vazi.

7 examinados em Matto Grosso (Porto Esperança). Apenas um apresentava microfilarias no sangue, mas não foram encontrados os adultos.

1 examinado em S. Paulo (Rio Claro) que não apresentava filarideos.

Nestes 20 exemplares de jacaré examinados um estava parasitado com $O$. bacillaris $(5 \%)$ e 5 com $M$. vazi ( $20 \%)$.

\section{BIBLIOGRAPHIA}

BAYLIS, 1924.-The male of Micropleura vivipara (Nematoda). Mag. Nat. Hist. Ser. 9, v. 13, p. 1991.

BAYLIS \& DAUBNEY, 1922.-Report on the parasitic nematodes in the Collection of the Zoological Survey of India. Mem. Ind. Mus., v. 7, p. 263.

BAYLIS \& DAUBNEY, 1926. - A synopsis of the families of genera of nematoda. 
V. LINSTOW, 1906.-Parasites from the Gharial (Gavialis gangeticus Geoffr.) J. of Proc. As. Soc. Beng. v. 2, p. 269.

MAPLESTONE, 1930.-Parasitic nematodes obtained from animals dying in the Calcutta Zoological Gardens. Rec. Ind. Mus. v. 23, p. 385 .

MOLIN, 1858.-Versuch einer Monographie der Filarien. Sitzungsb. K. Ak d. Wissench. Bd. 28, s. 365 .

PANDIT, PANDIT \& IYER, 1929.-A new filarid in Calotes versicolor. Conospiculum guindiensis. n. g., n. sp. Ind. J. Med. Res. v. 16, p. 954.

SEURAT, 1917.-Filaires des reptiles et des batraciens. Bull. Soc. Hist. Nat. de l'Afr. du Nord. T. 8, no. 9, 236.

YORKE \& MAPLESTONE, 1926.-The Nematode parasites of vertebrates.

\section{EXPLICAÇ̃̃O DAS ESTAMPAS XXXI-XXXIV}

\section{ESTAMPA XXXI}

Fig. 1-Oswaldofilaria bacillaris.-Extremidade cephalica da femea.

Fig. 2-Oswaldofilaria bacillaris.-Metade anterior do corpo da femea.

Fig. 3-Oswaldofilaria bacillaris.-Esphincter do ovejector.

\section{ESTAMPA XXXII}

Fig. 4-Oswaldofilaria bacillaris.-Extremidade posterior da femea.

Fig. 5-Oswaldofilaria bacillaris.-Cauda do macho.

Fig. 6-Micropleura vazi.-Extremidade anterior da femea jovem.

Fig. 7-Micropleura vazi.-Extremidade cephalica da femea jovem.

Fig. 8-Micropleura vazi.-Extremidade distal do ovario.

Fig. 9-Micropleura vazi.-Extremidade posterior da femea.

\section{ESTAMPA XXXIII}

Fig. 10-Oswaldofilaria bacillaris.-Espiculo.

Fig. 11-Micropleura vazi.-Evolução das larvas.

Fig. 12-Micropleura vazi.-Macho total.

\section{ESTAMPA XXXIV}

Fig. 13-Micropleura vazi.-Femea total.

Fig. 14-Micropleura vazi.-Extremidade cephalica do macho.

Fig. 15-Micropleura vazi.-Extremidade caudal do macho. 

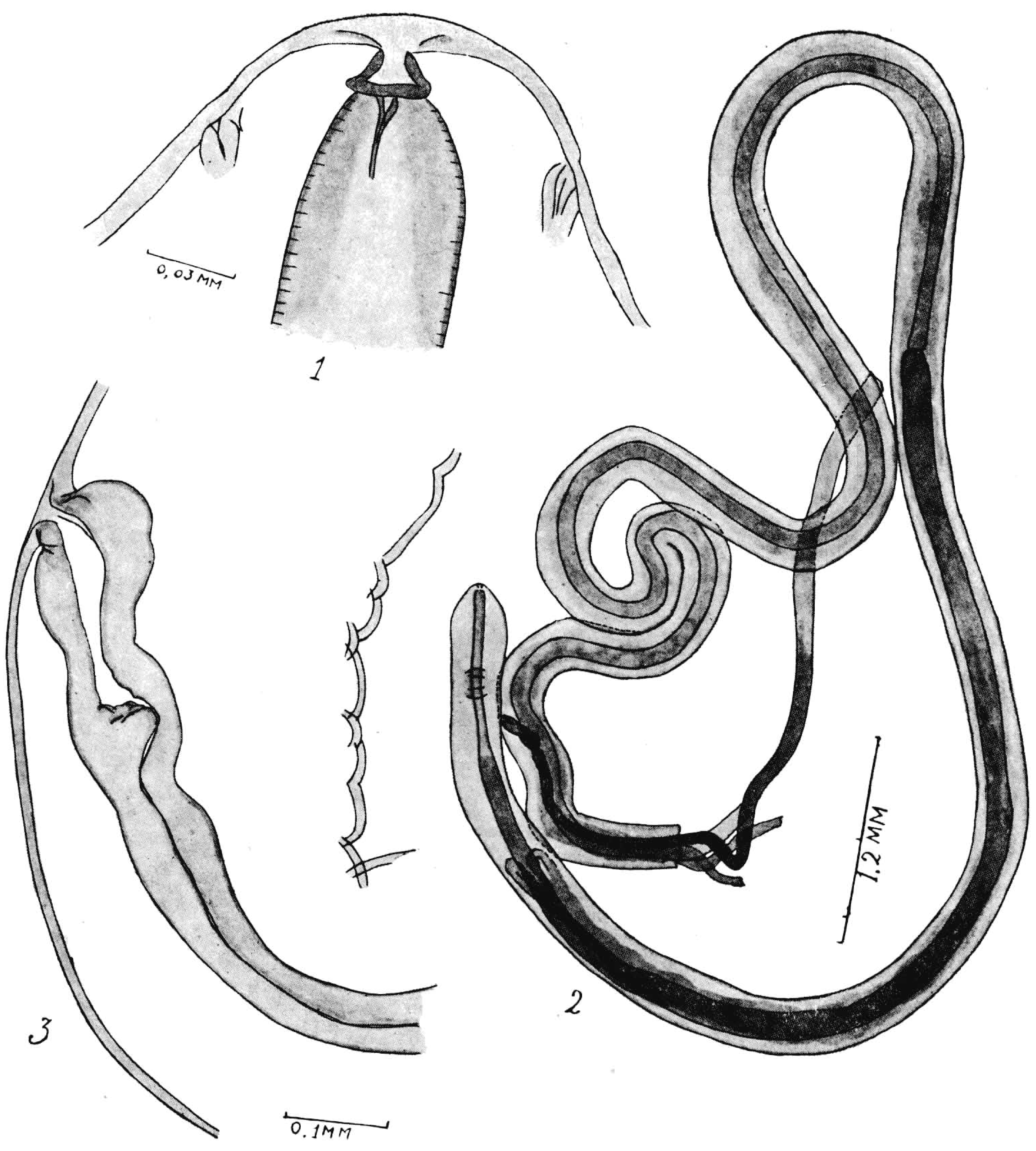

Dr. Lauro Travassos : Sobre os filarideos dos crocodillos sul-americanos. 


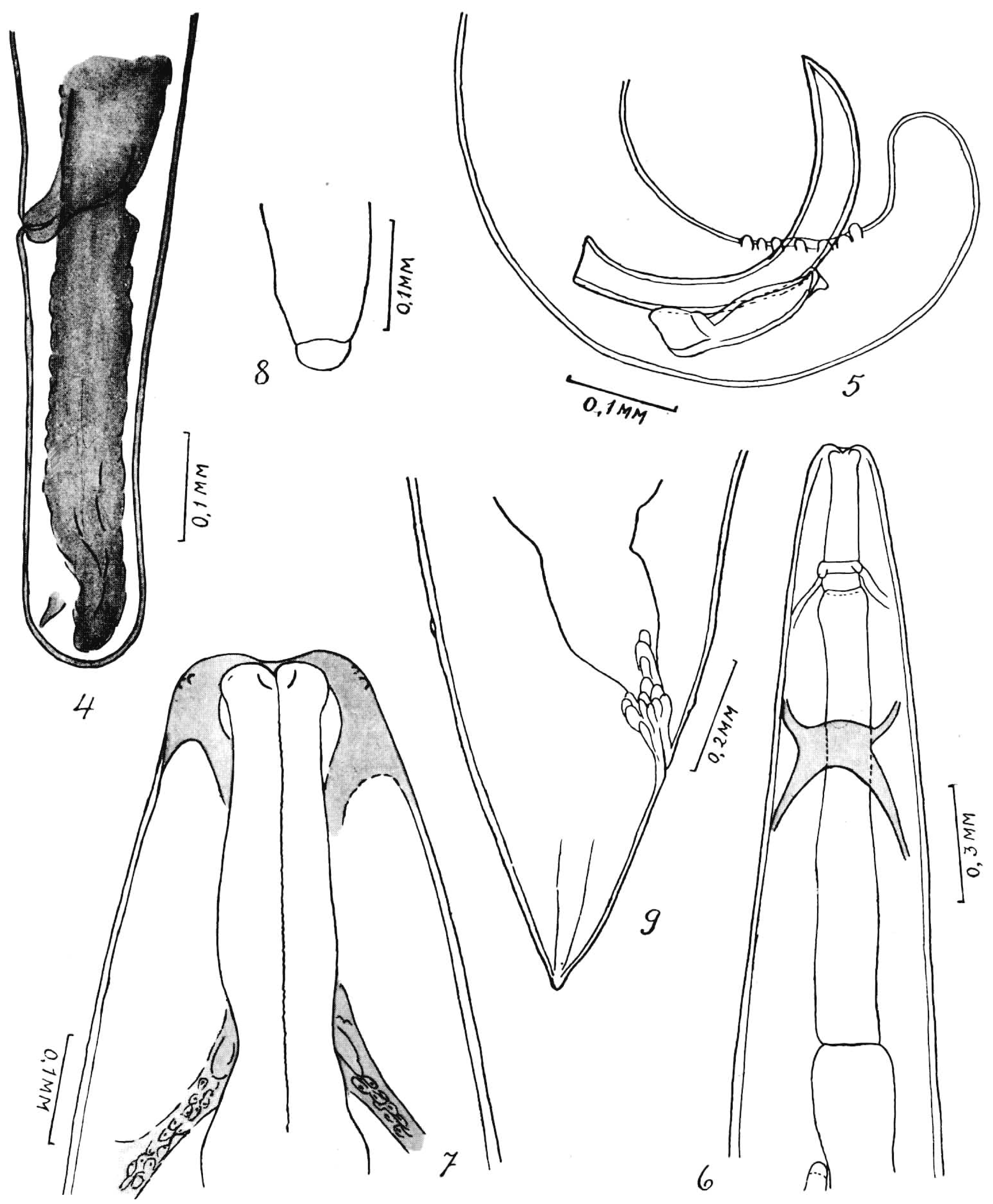

Dr. Lauro Travassos : Sobre os filarideos dos crocodillos sul-americanos. 


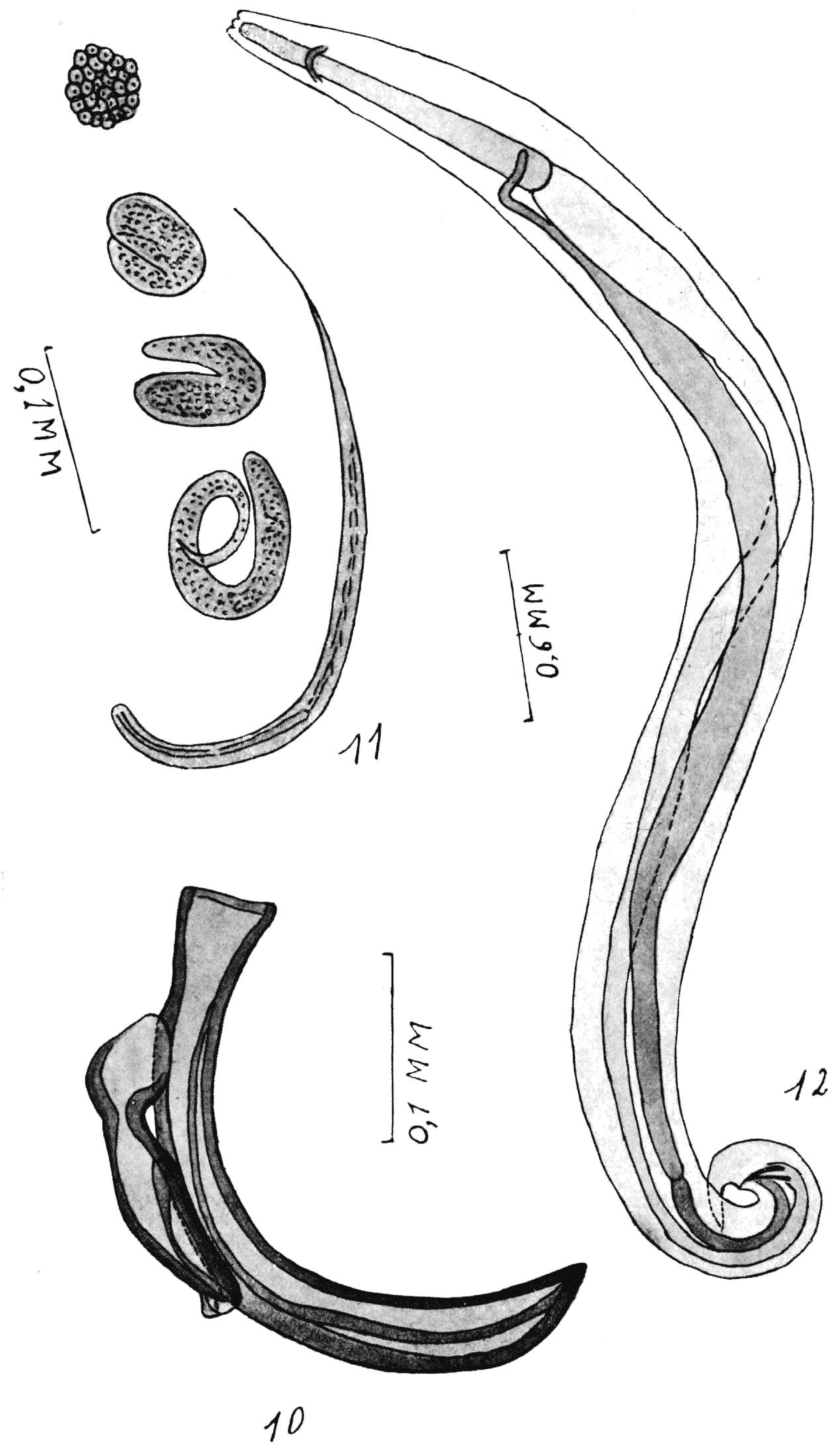

Dr. Lauro Travassos : Sobre os filarideos dos crocodillos sul-americanos. 

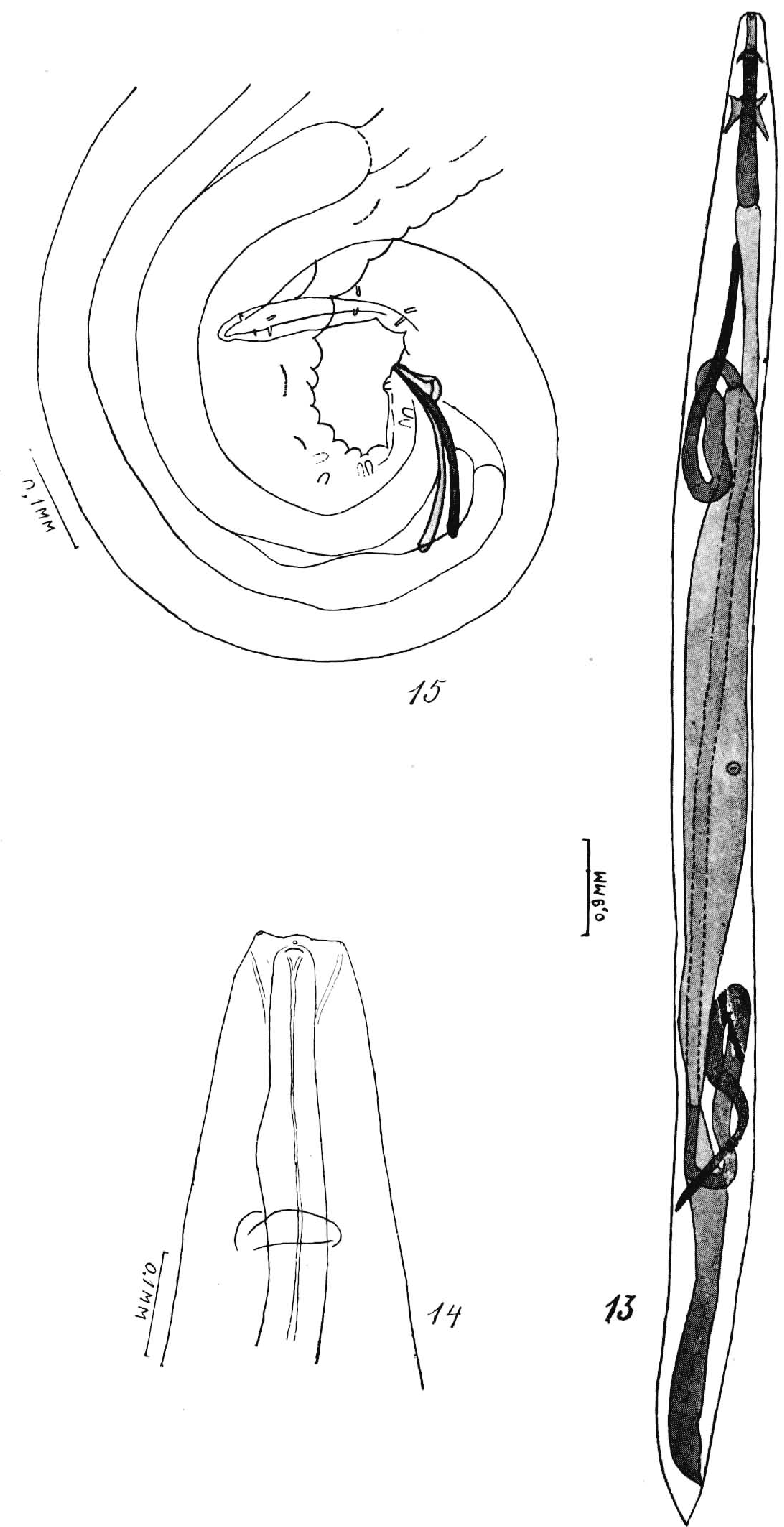

Dr. Lauro Travassos : Sobre os filarideos dos crocodillos sul-americanos. 